\title{
Eficiências Reprodutiva e Produtiva em Vacas das Raças Gir, Holandês e Cruzadas Holandês x Zebu ${ }^{1}$
}

\author{
José Domingos Guimarães ${ }^{2}$, Nadja Gomes Alves ${ }^{3}$, Eduardo Paulino da Costa ${ }^{2}$, \\ Márcio Ribeiro Silva ${ }^{4}$, Flávio Marcos Juqueira Costa ${ }^{4}$, Belmiro Zamperlini ${ }^{5}$
}

\begin{abstract}
RESUMO - Para avaliar a eficiência reprodutiva e sua relação com características produtivas e composição genética de um rebanho, criado em condições de clima tropical, foram utilizadas 113 vacas, distribuídas em sete grupamentos genéticos, como se segue: 1/2, 3/4, 7/8, 9/16 e 15/16 Holandês x Zebu, Holandês-PB-PC e Gir. As varáveis estudadas foram: idade ao parto, estação de parição, intervalo do parto ao primeiro serviço, intervalo do primeiro serviço ao serviço fértil, número de serviços, período de serviço, intervalo de partos e eficiência reprodutiva. Com relação a características produtivas, avaliou-se o período de lactação e produção de leite por ordem de lactação e grupamentos genéticos do rebanho. Foi utilizado o programa estatístico SAEG, realizando-se as análises de regressões múltiplas, de variância e de comparação de médias pelo teste de Tukey a 5\% de probabilidade de erro. A eficiência reprodutiva foi influenciada por idade ao parto, período de lactação, intervalo de partos e composição genética das vacas, porém não o foi pela produção de leite e estação do ano. Os índices produtivos não foram influenciados pela estação de parição, mas sim pela composição genética dos animais. Neste estudo, os índices reprodutivos mostraram-se satisfatórios, os animais zebuínos apresentaram os menores índices produtivos e os taurinos, a menor eficiência reprodutiva, provavelmente pela maior sensibilidade às condições de manejo.
\end{abstract}

Palavras-chave: bovinos, eficiência reprodutiva, produção de leite

\section{Reproductive and Productive Efficiencies in Holstein and Holstein x Zebu Cows Crossbreds}

\begin{abstract}
To evaluate the reproductive efficiency and its relationship with productive traits and genetic composition of a herd, in tropical climate, 113 cows were ssigned to seven crossbreeding levels: 1/2, 3/4, 7/8, 9/16 and 15/16 Holstein x Zebu, crossbreed pure Holstein and Gir. The studied traits were: age at calving, calving season, interval from calving to first service, interval from first service to fertile service, number of services, service period, interval calving, reproductive efficiency, lactation period, milk production by lactation order and genetic groups of the herd. Analyses of multiple regression, variance and comparison of averages using Tukey test were performed in statistical software SAEG. The reproductive efficiency was influenced by the age at calving, lactation period, calving interval and genetic composition of the cows, but it was not influenced by milk production and season. The productive indexes were not influenced by the calving season, but were influenced by the genetic composition of the animals. In this study, the reproductive indexes were satisfactory, the zebu cows presented the smallest productive indexes and the taurine ones the smallest reproductive efficiency, probably by larger sensibility to handling.
\end{abstract}

Key Words: bovine, milk production, reproductive efficiency

\section{Introdução}

A eficiência reprodutiva é um dos mais importantes segmentos para o bom desempenho da atividade pecuária, a qual pode, teoricamente, atingir valores próximos a 95\%. Entretanto, nas condições brasileiras, verificam-se uma taxa de desfrute de $18 \%$ e uma eficiência reprodutiva de 50\% (Azevedo et al., 1989).

A pecuária de leite nacional caracteriza-se basicamente por bovinos mestiços, originados do cruza- mento de raças européias (particularmente Holandês). Os cruzamentos entre raças podem gerar animais de maior produtividade, por causa da combinação das características das raças utilizadas e também do fenômeno do "vigor híbrido", ou heterose. Diaz Gonzalez (1991) afirmou que animais com grau de sangue zebuíno têm maior eficiência na utilização dos volumosos, maior adaptabilidade às condições adversas e, conseqüentemente, tendem a apresentar melhor índice reprodutivo em condições tropicais.

\footnotetext{
${ }^{1}$ Apoio financeiro da FAPEMIG - Fundação de Amparo à Pesquisa do Estado de Minas Gerais.

2 Universidade Federal de Viçosa - Departamento de Veterinária. E.mail: epcosta@mail.ufv.br. Avenida P. H. Rolfs, s/n - Campus Universitário - Viçosa, MG - Cep: 36571-000.

${ }^{3}$ Aluna de Doutorado - Universidade Federal de Viçosa - Departamento de Zootecnia - Viçosa - MG. E.mail: nadja10@globo.com

${ }^{4}$ Médico-Veterinário.

5 Universidade Federal de Viçosa - Departamento de Zootecnia.
} 
A produção de leite é utilizada tradicionalmente como o mais importante parâmetro em programas de seleção de rebanhos leiteiros. Entretanto, vários estudos têm evidenciado que a eficiência reprodutiva declina com o aumento da produção leiteira (Campos et al., 1994, 1995), sendo necessário estabelecer a relação genética entre ambas (Raheja et al., 1989). Apesar desse aspecto, Raheja et al. (1989) não evidenciaram tal relação no estudo de rebanhos bovinos constituídos de vacas Holandesas. Segundo Short et al. (1990), citados por Campos et al. (1994), uma relação indesejável que talvez possa existir provoca poucos efeitos nos custos totais, pela compensação da maior produção.

Tem sido verificado que, quanto menor a idade das vacas ao primeiro parto, menor a produção do animal (Heinrichs \& Vazquez-Anon, 1993). Segundo Gill \& Allaire (1976), a idade ideal ao primeiro parto para atingir a eficiência máxima durante a vida útil da vaca é de 22,5 a 23,5 meses. Entretanto, a idade ao primeiro parto encontrada por Nobre (1983) foi de 33,9 ; 44,9; e 39,0 meses para animais $1 / 2$ e 3/4 Holandês x Zebu (HZ) e Holandeses puros por cruza (HPC), respectivamente, sendo essa idade influenciada pelo grau de sangue. Contrariamente, Campos (1987) não observou influência da estação de nascimento ou grau de sangue, verificando uma média de 41,1 meses.

O intervalo do parto ao primeiro serviço tem sido, em média, de 70 a 90 dias (Silva et al., 1992). Contudo, maiores intervalos ocorrem nas fêmeas de primeiro e segundo partos, devido ao estresse da lactação e ao desenvolvimento físico ainda incompleto. Adicionalmente, as condições corporais no pós-parto e o grau de sangue podem influenciar a duração desse intervalo (Dias, 1983), apesar de outros pesquisadores não terem constatado o efeito do grau de sangue (Raheja et al., 1989; Lobato, 1992).

Segundo Smith \& Legates (1962), as vacas de alta produção têm mais dificuldade para conceber do que as de baixa produção. Esse aspecto influencia a produção total durante a vida da vaca, devido ao aumento do período de serviço, sendo a produção máxima obtida em vacas com período de serviço mínimo (Matsoukas $\&$ Fairchild, 1974). Tais achados, entretanto, contrastam com os verificados por Schaeffer \& Henderson (1972), Olds et al. (1979) e Lee et al. (1997), os quais observaram que, apesar do aumento no período de serviço, a cada estádio sucessivo de lactação a produção de leite total também aumenta.
Os estudos envolvendo a relação entre produção e eficiência reprodutiva devem considerar fatores ambientais, como o ano e a estação (Smith \& Legates, 1962). Spears et al. (1965) estimaram que o efeito do ano na fertilidade responde por $1,4 \%$ do total, enquanto Olds et al. (1966) mostraram que esse componente de variação foi de $2,0 \%$. Têm sido encontrados efeitos do ano sobre idade à primeira parição (Nobre, 1983), ano de parto (Nobre, 1983; Silva, 1992) e estação do ano sobre o intervalo de partos (Dias, 1983; Silva et al., 1992) e sobre o período de serviço (Silva et al., 1992). Apesar dessas observações, Matsoukas \& Fairchild (1974) verificaram que o mês e o ano de parto não tiveram nenhum efeito sobre as medidas de eficiência reprodutiva.

Uma influência significativa do mês ou estação de parição sobre a produção de leite foi encontrada por Nobre (1983), ressaltando-se que as produções das lactações iniciadas no outono-inverno apresentaram períodos de lactação mais longos e maior produção de leite do que as iniciadas na primavera-verão, provavelmente devido ao reflexo de manejo. Essas observações foram corroboradas por Teodoro et al. (1994), também em rebanhos da raça Holandês e por Souza et al. (1995) em rebanhos da raça Gir. Entretanto, Campos (1987) e Lopes et al. (1996) não verificaram nenhum efeito da estação de parição sobre a produção, em rebanhos submetidos a manejo intensivo de produção de leite.

Quanto aos aspectos genéticos, têm sido encontrados resultados conflitantes sobre a influência do grau de sangue na produção. Assim, alguns pesquisadores verificaram que os animais mestiços tiveram maior produção (Nobre, 1983; Campos, 1987; Lobato, 1992), enquanto em outras pesquisas não se evidenciou efeito do grau de sangue nesta variável (Barbosa et al., 1994b; Teodoro et al., 1994).

Segundo Barbosa et al. (1994a), o período de lactação ideal foi de 305 dias, na expectativa de se obter uma lactação por ano. Entretanto, a duração do período de lactação pode ser influenciada por vários fatores, o que, conseqüentemente, pode influenciar a produção leiteira. Nobre (1983) constatou elevado coeficiente de correlação entre a duração da lactação e o total de leite produzido. Estudando o efeito do grau de sangue sobre o período de lactação, Nobre (1983) e Thaler Neto et al. (1995) não verificaram nenhuma influência. Entretanto, várias pesquisas têm evidenciado o efeito dos diferentes grupos genéticos na duração da lactação, sendo esta geralmente maior nos animais 
puros de origem, quando comparados com os puros por cruza (Campos et al., 1987; Lobato, 1992; Teodoro et al., 1994; Barbosa et al., 1994a).

As contradições nos resultados apresentados nesta revisão de literatura justificam-se pelas diferentes variáveis que ocorreram, particularmente, em cada rebanho estudado. Objetivou-se neste experimento verificar quais são os índices reprodutivos (idade ao parto, estação de parição, intervalo do parto ao primeiro serviço, número de serviços, período de serviço e intervalo de partos) e produtivos (produção de leite e período de lactação) que influenciaram a eficiência reprodutiva dos animais de diferentes cruzamentos absorventes Holandês x Zebu.

Objetivou-se também estabelecer as relações entre as características produtivas e reprodutivas dos animais, bem como verificar quais são os graus de sangue que mais se adaptam às condições de criação local.

\section{Material e Métodos}

Foram utilizados dados produtivos e reprodutivos do rebanho proveniente do Setor de Bovinocultura de Leite, do Departamento de Zootecnia da Universidade Federal de Viçosa, em Viçosa, Minas Gerais. O estudo compreendeu registros de controles reprodutivo e leiteiro no período de abril de 1986 a janeiro de 1997, totalizando 113 vacas, distribuídas em grupos de acordo com a composição genética, ou seja: 1/2 (10), 3/4 (33), 7/8 (23), 9/16 (7) e 15/16 (11) Holandês X Zebu, HPC (25) e Gir (4).

As vacas foram criadas em regime de semiestabulação, em pastagem predominantemente de Brachiaria decumbens, suplementadas com silagem de milho e ração balanceada, de acordo com a produção leiteira, e ordenhadas duas vezes ao dia.

Foram avaliados os seguintes parâmetros: intervalo do parto ao primeiro serviço (dias), número de serviços, período de serviço (dias), intervalo de partos (dias), estação de parição (períodos seco e chuvoso), eficiência reprodutiva (EF), produção de leite por lactação $(\mathrm{kg})$ e período de lactação (dias). A eficiência reprodutiva foi definida segundo os cálculos: $\mathrm{EF}=$ número de partos $\mathrm{x}$ ( 365 x 100/intervalo do primeiro ao último parto), sendo o intervalo especificado em dias. A estação seca de parição foi definida como de maio a outubro e a chuvosa, de novembro a abril.

Foram realizadas análises pelo sistema SAEG, compreendendo avaliações univariadas e estatísticas com quebras para obtenção das médias e desvios- padrão das variáveis estudadas. Foi realizada a análise de variância geral, tomando-se como variável dependente a produção de leite e, como variáveis independentes, o grau de sangue, a idade ao parto e a estação do ano, visando avaliar o efeito dessas variáveis na produção de leite. Quando verificado efeito significativo pelo teste $\mathrm{F}$, realizou-se a comparação de médias pelo teste de Tukey a 5\% de probabilidade de erro.

Empregou-se ainda a análise de variância geral, tomando como variável independente o grau de sangue e, como variáveis dependentes, a eficiência reprodutiva, a produção de leite por lactação, o período de lactação, o intervalo de partos, o período de serviço, o intervalo do parto ao primeiro serviço e o número de serviços por prenhez. Quando verificado efeito significativo pelo teste F, realizou-se a comparação de médias pelo teste de Tukey a $5 \%$ de probabilidade de erro.

Empregou-se, também, a análise de regressão múltipla (stepwase), tendo como variável dependente a eficiência reprodutiva e, como variáveis independentes, o grau de sangue, a idade ao parto, a estação de parição, o número de serviços por prenhez, o intervalo do parto ao primeiro serviço, o período de serviço, o intervalo de partos, a produção de leite e o período de lactação.

Foram efetuadas as correlações simples de Pearson de todas as variáveis entre si, a $5 \%$ de probabilidade de erro.

\section{Resultados e Discussão}

As médias e os desvios-padrão referentes aos parâmetros avaliados do rebanho estudado são apresentados na Tabela 1.

Verificou-se que a eficiência reprodutiva foi influenciada pela idade ao parto $(\mathrm{P}<0,01)$ e pelo período de lactação $(\mathrm{P}<0,05)$, no entanto não o foi $(\mathrm{P}>0,05)$ pela produção de leite, concordando com os achados de Raheja et al. (1989) e Dong \& Van Vleck (1989) e contrariando os relatos de Campos et al. (1994) e Campos et al. (1995). Como não se trata de um rebanho de elevada produção, esse aspecto provavelmente não tenha contribuído de forma a interferir na eficiência reprodutiva.

A eficiência reprodutiva correlacionou-se negativamente com o número de inseminações $(\mathrm{r}=-0,3162)$ e período de serviço $(r=-0,3162)$. Essa correlação 
Tabela 1 - Parâmetros reprodutivos e produtivos avaliados em vacas com diferentes graus de sangue Holandês $x$ Zebu, no período de abril de 1986 a janeiro de 1997

Table 1 - Productive and reproductive parameters in cows with different crossbreeding Holstein $x$ Zebu levels, in the period from abril, 1986 to january, 1997

\begin{tabular}{lccc}
\hline $\begin{array}{l}\text { Variável } \\
\text { Trait }\end{array}$ & $\begin{array}{c}\text { Número } \\
\text { Number }\end{array}$ & $\begin{array}{c}\text { Médias do rebanho } \\
\text { Herd averages }\end{array}$ & $\begin{array}{c}\text { Desvio-padrão } \\
\text { Standard deviation }\end{array}$ \\
\hline ER & 858 & 89,09 & 13,91 \\
IDP & 368 & $1.744,35$ & 708,55 \\
NIA & 301 & 1,74 & 1,20 \\
IPPS & 340 & 99,26 & 136,21 \\
IPSSF & 291 & 58,47 & 114,81 \\
PS & 297 & 158,21 & 184,29 \\
IP & 247 & 414,70 & 104,79 \\
PLE & 292 & $3.809,98$ & $1.716,86$ \\
PLA & 292 & 282,15 & 87,19 \\
\hline
\end{tabular}

ER: eficiência reprodutiva (\%), IDP: idade ao parto (dias), NIA: número de inseminações artificiais, IPPS: intervalo do parto ao primeiro serviço (dias), IPSSF: intervalo do primeiro serviço ao serviço fértil (dias), PS: período de serviço (dias), IP: intervalo de partos (dias), PLE: produção de leite $(\mathrm{kg})$ e PLA: período de lactação (dias).

ER: reproductive efficiency (\%), IDP: age at calving (days), NIA: number of artificial inseminations, IPPS: interval from calving to first service (days), IPSSF: interval from first service to fertile service (days), PS: service period (days), IP: calving interval (days), PLE: milk production (kg), PLA: lactation period (days).

negativa pode ser justificada pelo fato de serem os parâmetros que influenciam indiretamente as variáveis consideradas no cálculo da eficiência reprodutiva. Os dados apresentados na Tabela 1, relacionados ao intervalo primeiro serviço-serviço fértil, evidenciam prováveis deficiências no manejo de detecção do estro. Outro aspecto a considerar é a possibilidade da ocorrência de anestro devido à instalação de infecções uterinas pós-inseminação. Ressalta-se, ainda, que alguns animais apresentaram intervalo do primeiro serviço ao serviço fértil demasiadamente longo, o que contribuiu para esse intervalo médio de 58 dias. Tal condição é caracterizada pelo elevado desvio-padrão (114 dias).

A estação do ano não apresentou correlação com nenhum dos índices estudados, corroborando os achados de Campos (1987), que trabalhou com mestiças 1/4 a 15/16 Holandês; e de Lopes et al. (1996), trabalhando com vacas Holandesas PO e PC. Da mesma forma, Matsoukas \& Fairchild (1974) não encontraram efeito do mês e do ano de parto nas medidas de eficiência reprodutiva em rebanhos das raças Holandês e Jersey. É provável que, assim como no presente experimento, esses pesquisadores tenham estudado rebanhos com manejo mais adequa- do, principalmente no que diz respeito à nutrição, já que, segundo Nobre (1983), essa influência parece estar estreitamente relacionada com a variação das condições climáticas de um ano para outro, notadamente a disponibilidade de alimentos e práticas de manejo em geral. Entretanto, Nobre (1983), trabalhando com vacas 1/2, 3/4 Holandês e Holandês-PC; Teodoro et al. (1994), com rebanhos da raça Holandesa; e Souza et al. (1996), com rebanhos da raça Gir, verificaram que as lactações iniciadas no outono-inverno (época seca) foram maiores do que as com início na primavera-verão, porque os rebanhos foram suplementados durante o outono-inverno e, também, porque nessa época as condições climáticas são mais favoráveis a esses animais. Alguns pesquisadores também encontraram efeito da estação do ano sobre o intervalo de partos (Dias, 1983; Silva et al., 1992) e o período de serviço (Silva et al., 1992), o que poderia ser, provavelmente, por condições alimentares mais deficientes em determinadas épocas do ano. Além disso, os referidos pesquisadores trabalharam com animais puros, que são mais sensíveis às condições de manejo alimentar.

O grau de sangue influenciou $(\mathrm{P}<0,05)$ a produção de leite. As maiores produções foram apresentadas pelos grupos $7 / 8$ e 3/4, enquanto a menor ocorreu com os animais do grupo Gir (Tabela 2). Essa maior produção leiteira dos animais oriundos de cruzamentos absorventes Holandês x Zebu também foi verificada por outros pesquisadores (Nobre, 1983; Campos, 1987; Lobato, 1992). É provável que isso seja devido à maior adaptação dos mestiços ao ambiente, conforme justificado por Nobre (1983). Entretanto, Barbosa et al. (1994b) não observaram efeito do grau de sangue sobre a produção de leite. Da mesma forma, Teodoro et al. (1994) verificaram igualdade entre os grupos genéticos estudados, em razão do ajuste das produções para 305 dias de lactação, o que remove a possível variação entre os grupos genéticos.

Verificou-se que a eficiência reprodutiva foi influenciada pelo grau de sangue e intervalo de partos $(\mathrm{P}<0,05)$, apresentando com este último uma correlação negativa ( $\mathrm{r}=-0,5349)$, que é procedente, já que ambas são variáveis interdependentes. Adicionalmente, o incremento no período de serviço $(\mathrm{r}=0,40737)$ e no número de serviços $(\mathrm{r}=0,46124)$ provocou aumento no intervalo de partos, comprometendo a eficiência reprodutiva.

No presente estudo não houve influência $(\mathrm{P}>0,05)$ 
do grau de sangue no período de serviço entre os diferentes cruzamentos absorventes, em que existia sangue Holandês. Da mesma forma, não foi verificada influência $(\mathrm{P}>0,05)$ do grau de sangue sobre o intervalo de partos, embora o período de serviço da raça Gir tenha sido maior $(\mathrm{P}<0,05)$ do que o das vacas dos grupos $1 / 2$ e 3/4 Holandês x Zebu (Tabela 2). Esse maior período pode ser devido ao fato de os animais da raça Gir terem apresentado maior intervalo do parto ao primeiro serviço, já que houve correlação positiva $(r=0,68803)$ entre essas duas variáveis. Tais resultados concordam com os de Nobre (1983), Lobato (1992) e Dias (1993), os quais trabalharam com diferentes cruzamentos Holandês x Zebu. Entretanto, os referidos pesquisadores não estudaram animais da raça Gir. É provável que o manejo alimentar adequado do rebanho pesquisado tenha favorecido para que não houvesse influência dos diferentes grupos genéticos onde existia sangue Holandês, concordando com as afirmações de Campos (1987).

Considerando o intervalo do parto ao primeiro serviço, não foram observadas diferenças $(\mathrm{P}>0,05)$ entre os diferentes cruzamentos Holandês x Zebu, em comparação com os animais HPC (Tabela 2). Esses resultados contrastam com os de Dias (1983), que observou menor intervalo em vacas $1 / 2$ sangue Holandês x Zebu, indicando a influência de heterose. Apesar de não-significativa, foi verificada, no presente experimento, a tendência de menor intervalo também nos animais $1 / 2$ sangue. Lobato (1992) observou menor intervalo do parto ao primeiro serviço em animais 9/16 e 7/8 Holandês x Zebu. Contudo, essa pesquisadora estudou somente esses cruzamentos, além do Holandês-PC.

A produção leiteira não foi influenciada $(\mathrm{P}>0,05)$ pela estação de parição, entretanto a ordem de parto afetou $(\mathrm{P}<0,01)$ esta variável. Esses achados concordam com os de Lopes et al. (1996), os quais observaram superioridade das vacas de segunda e demais ordens, quanto às produções inicial e máxima, o que poderia ser explicado pelo fato de essa classe possuir animais mais próximos da maturidade fisiológica. Corroborando esses resultados, Campos et al. (1987) observaram aumento de 0,45 litro de leite por vaca em lactação para cada aumento na ordem média de partos. A produção de leite por lactação apresentou correlação positiva $(\mathrm{r}=0,85011)$ com o período de lactação. Dessa forma, é provável que o curto período de lactação em animais puros Gir (6 a 7,7 meses) tenha influenciado os resultados.

O período de lactação foi influenciado pelos diferentes graus de sangue $(\mathrm{P}<0,01)$, sem que, no entanto, apresentasse diferenças na comparação das médias (Tabela 2), corroborando os achados de Nobre (1983) e Thaler Neto et al. (1995). Entretanto, outros pesquisadores (Lobato, 1992; Teodoro et al., 1994) observaram diferenças significativas entre essas duas variáveis. Segundo Campos (1987), o período de lactação aumenta à medida que também aumenta a proporção de sangue Holandês. Essa afirmação corrobora a tendência encontrada neste experimento, embora sem diferença significativa. Os animais da raça Gir apresentaram tendência de menor período, enquanto as vacas $3 / 4$ e $7 / 8$ Holandês x Zebu e Holandês-PC

Tabela 2 - Eficiência reprodutiva, produção de leite por lactação, período de lactação, período de serviço e intervalo do parto ao primeiro serviço em função dos diferentes graus de sangue

Table 2 - Reproductive efficiency, milk production per lactation, lactation period, service period, interval from calving to first service, in relation to the different crossbreeding levels

\begin{tabular}{|c|c|c|c|c|c|c|c|c|c|c|}
\hline \multirow{2}{*}{$\begin{array}{l}\text { GS } \\
\text { HPC }\end{array}$} & \multicolumn{2}{|c|}{ ER } & \multicolumn{2}{|c|}{ PLE } & \multicolumn{2}{|c|}{ PLA } & \multicolumn{2}{|c|}{ PS } & \multicolumn{2}{|c|}{ IPPS } \\
\hline & $(170)$ & $84,31^{\mathrm{c}}$ & $(056)$ & $3864,89^{a, b, c}$ & $(056)$ & $284,25^{\mathrm{a}}$ & $(057)$ & $157,46^{\mathrm{a}, \mathrm{b}}$ & $(057)$ & $80,11^{b}$ \\
\hline $15 / 16$ & (060) & $90,57^{b, c}$ & (020) & $3579,70^{\mathrm{a}, \mathrm{b}, \mathrm{c}}$ & $(020)$ & $268,25^{a}$ & (017) & $149,06^{\mathrm{a}, \mathrm{b}}$ & (017) & $74,91^{\mathrm{b}}$ \\
\hline $7 / 8$ & (140) & $100,30^{\mathrm{a}}$ & $(035)$ & $4244,95^{\mathrm{a}}$ & $(035)$ & $289,94^{\mathrm{a}}$ & $(035)$ & $158,74^{\mathrm{a}, \mathrm{b}}$ & $(035)$ & $65,23^{\mathrm{b}}$ \\
\hline $3 / 4$ & $(270)$ & $86,86^{\mathrm{b}, \mathrm{c}}$ & (076) & $4000,69^{\mathrm{a}, \mathrm{b}}$ & $(076)$ & $298,75^{\mathrm{a}}$ & (084) & $142,56^{\mathrm{b}}$ & $(084)$ & $84,80^{\mathrm{b}}$ \\
\hline $9 / 16$ & (070) & $91,90^{\mathrm{a}, \mathrm{b}}$ & (043) & $3162,17^{\mathrm{b}, \mathrm{c}, \mathrm{d}}$ & (043) & $239,21^{\mathrm{a}}$ & (043) & $174,35^{\mathrm{a}, \mathrm{b}}$ & $(044)$ & $136,52^{\mathrm{ab}}$ \\
\hline $1 / 2$ & $(050)$ & $100,58^{\mathrm{a}}$ & (016) & $2681,63^{\mathrm{c}, \mathrm{d}}$ & (016) & $231,94^{\mathrm{a}}$ & (015) & $77,87^{\mathrm{b}}$ & (015) & $48,95^{\mathrm{b}}$ \\
\hline Gir & (030) & $90,45^{a, b, c}$ & (008) & $1427,06^{\mathrm{d}}$ & (008) & $189,50^{\mathrm{a}}$ & (008) & $337,88^{a}$ & (010) & $212,30^{\mathrm{a}}$ \\
\hline
\end{tabular}

Médias seguidas de letras iguais na mesma coluna não diferem entre si $(P>0,05)$. GS: grau de sangue. ER: eficiência reprodutiva (\%), PLE: produção de leite por lactação (kg), PLA: período de lactação (dias), PS: período de serviço (dias), IPPS: intervalo do parto ao primeiro serviço (dias) e ( ): número de repetições.

Average values followed by the same letters in the same column do not differ (P>.05). GS: crossbreeding level. ER: reproductive efficiency (\%), PLE: Milk production per lactation (kg), PLA: lactation period (days), PS: service period (days), IPPS: Interval from calving to first service (days) and (): number of repetitions. 
tiveram comportamento inverso. O período de lactação não foi influenciado pela ordem de parto ( $\mathrm{P}>0,05)$, o que foi também observado por Barbosa et al. (1994a). Entretanto, Campos (1987) verificou maiores períodos de lactação em vacas mais jovens, possivelmente devido aos maiores períodos de serviço destas.

\section{Conclusões}

Animais da raça Gir apresentaram menor produção de leite quando comparados com os HPC. Os mestiços exibiram valores intermediários, com tendência a se comportarem de acordo com a maior ou a menor proporção de sangue Gir.

Houve tendência de maior produção de leite e período de lactação mais extenso em animais 7/8 e 3/4 Holandês x Zebu, talvez pela proporção de Holandês, associada à maior adaptabilidade desses a a ambiente, em comparação com os animais puros.

A eficiência reprodutiva foi influenciada pela proporção de Holandês ou Zebu, ocorrendo menor eficiência em animais Holandês-PC, provavelmente pela maior sensibilidade às condições de manejo.

Os animais $1 / 2$ sangue Holandês x Zebu apresentaram menor período de serviço e intervalo do parto ao primeiro serviço do que vacas da raça Gir.

\section{Agradecimento}

À FAPEMIG, pelo financiamento integral deste estudo, e ao Setor de Bovinocultura do Departamento de Zootecnia da Universidade Federal de Viçosa, por possibilitar a análise dos registros de controle zootécnico do rebanho estudado.

\section{Literatura Citada}

AZEVEDO, N.A.; COELHO, E.N.; REFHELD, O.A.M. et al. Reprodução - Importante segmento na produção animal. Informe Agropecuário, v.14, n.163, p.123-132, 1989.

BARBOSA, S.B.P.; MANSO, H.C.; SILVA, L.O.C. Estudo do período de lactação em vacas holandesas no Estado de Pernambuco. Revista da Sociedade Brasileira de Zootecnia, v.23, n.3, p.465-475, 1994a.

BARBOSA, S.B.P.; MILAGRES, J.C.; REGAZZI, A.J. et al. Estudo da produção de leite em rebanhos holandeses, no Estado de Pernambuco. Revista da Sociedade Brasileira de Zootecnia, v.23, n.3, p.422-432, 1994b.

CAMPOS, J.M.S. Aspectos reprodutivos e produtivos em um sistema de produção de leite, na microrregião de Viçosa, Estado de Minas Gerais. Viçosa, MG: Universidade Federal de Viçosa, 1987. 109p. Dissertação (Mestrado em Zootecnia) Universidade Federal de Viçosa, 1987.
CAMPOS, M.S.; WILCOX, C.M.; BECERRIL, C.M. et al. Genetic parameters for yield and reproductive traits of Holstein and Jersey cattle in Florida. Journal of Dairy Science, v.77, p.867-873, 1994.

CAMPOS, M.S.; WILCOX, C.J.; SPREEN, T.H. Effects of interrelationships of production and reproduction on net returns in Florida. Journal of Dairy Science, v.78, p.704-709, 1995.

DIAS, J.P. Eficiência reprodutiva no rebanho de vacas puras e mestiças holandesas da UFV. Viçosa/MG: Universidade Federal de Viçosa, 1983. 61p. Dissertação (Mestrado em Zootecnia) - Universidade Federal de Viçosa, 1983.

DIÁZ GONZÁLEZ, F.H. Efeito da condição corporal de novilhas sobre a fertilidade, perfil metabólico pós-serviço e sobrevivência embrionária. Viçosa/MG: Universidade Federal de Viçosa, 1991. 118p. Tese (Doutorado em Zootecnia) - Universidade Federal de Viçosa, 1991.

DONG, M.C.; Van VLECK, L.D. Correlations among first and second lactation milk yield and calving interval. Journal of Dairy Science, v.72, n.3, p.678-684, 1989.

ERB, R.E.; GOODWIN, M.M.; MORRISON, R.A. et al. Lactation studies. 1. Effect of gestation. Journal of Dairy Science, v.35, p.224-233, 1952.

GARDNER, R.W.; SCHUH, J.D.; VARGUS, L.C. Accelerated growth and early breeding of Holstein heifers. Journal of Dairy Science, v.60, n.12, p.1941-1948, 1977.

GILL, G.S.; ALLAIRE, F.R. Relationship of age at first calving, days open, days dry, and herdlife to a profit function for dairy cattle. Journal of Dairy Science, v.59, n.6, p.11311139, 1976.

HEINRICHS, A.J.; VAZQUEZ-ANON, M. Changes in first lactation dairy herd improvement records. Journal of Dairy Science, v.76, p.671-675, 1993.

LEE, K.J.; VANRADEN, P.M.; NORMAN, H.D. et al. Relationship of yield during early lactation and days open during current lactation with 305-day yield. Journal of Dairy Science, v.80, p.771-776, 1997.

LEGATES, J.E. Genetic variation in services per conception and calving interval in dairy cattle. Journal of Animal Science, v.13, p.81-88, 1954.

LIN, C.Y.; ALLAIRE, F.R. Relative efficiency of selection methods for profit on dairy cows. Journal of Dairy Science, v.60, n.12, p.1970-1978, 1977.

LOBATO, V. Desempenho reprodutivo e produtivo de vacas mestiças leiteiras, submetidas a dois níveis nutricionais, nos períodos pré e pós-parto. Viçosa/MG: Universidade Federal de Viçosa, 1992. 73p. Dissertação (Mestrado em Zootecnia) - Universidade Federal de Viçosa, 1992.

LOPES, M.A.; NEIVA, R.S.; VALENTE, J. et al. Aplicação da função tipo gama incompleta no estudo da curva de lactação de vacas da raça holandesa, variedade preta e branca, mantidas em sistema intensivo de produção. Revista da Sociedade Brasileira de Zootecnia, v.25, n.6, p.1086-1101, 1996.

LOUCA A.; LEGATES, J.E. Production losses in dairy cattle due to days open. Journal of Dairy Science, v.51, n.4, p.573-583, 1968.

MATSOUKAS, J.; FAIRCHILD, T.P. Effects of various factors on reproductive efficiency. Journal of Dairy Science, v.58, n.4, p.540-544, 1974.

NOBRE, P.R.C. Fatores genéticos e de meio em características produtivas e reprodutivas do rebanho leiteiro da Universidade Federal de Viçosa, Estado de Minas Gerais. Viçosa, MG: Universidade Federal de Viçosa, 1983. 128p. Dissertação (Mestrado em Zootecnia) Universidade Federal de Viçosa, 1983. 
OLDS, D.; COOPER, T.; THRIFT, F.A. Relationships between milk yield and fertility in dairy cattle. Journal of Dairy Science, v.62, p.1140-1144, 1979.

RAHEJA, K.L.; BURNSIDE, E.B.; SCHAEFFER, L.R. Relationships between fertility and production in Holstein dairy cattle in different lactations. Journal of Dairy Science, v.72, p.2670-2678, 1989.

SCHAEFFER, L.R.; HENDERSON, C.R. Effects of days dry and days open on Holstein milk production. Journal of Dairy Science, v.55, n.1, p.107-112, 1972.

SILVA, H.M.; WILCOX, C.J.; THATCHER, W.W. et al. Factors affecting days open, gestation length, and calving interval in Florida dairy cattle. Journal of Dairy Science, v.75, p.288293, 1992.

SMITH, J.W.; LEGATES, J.E. Relation of days open and days dry to lactation milk and fat yields. Journal of Dairy Science, v.45, p.1192-1198.

SOUZA, E.M.; MILAGRES, J.C.; MARTINEZ, M.L. et al. Efeitos de fatores genéticos e de meio ambiente sobre a duração do período de lactação em rebanhos de Gir leiteiro. Revista da Sociedade Brasileira de Zootecnia, v.24, n.1, p.150-163, 1995.

SOUZA, E.M.; MILAGRES, J.C.; REGAZZI, A.J. et al. Efeitos de fatores genéticos e de meio ambiente sobre a produção de leite em rebanhos de Gir leiteiro. Revista da Sociedade Brasileira de Zootecnia, v.25, n.4, p.888-901, 1996.

SPEARS, J.R.; OLDS, D.; COOPER, T. Evaluation of sources of variance in dairy herd fertility. Journal of Dairy Science, v.48, p.90-92, 1965.
THALER NETO, A.; NEIVA, R.S.; OLIVEIRA, A.I.G. et al. Causas de variação do desempenho produtivo de rebanhos da raça holandesa, no estado de Santa Catarina - Período de lactação. Revista da Sociedade Brasileira de Zootecnia, v.24, n.1, p.117-125, 1995.

TEODORO, R.L.; MILAGRES, J.C.; CARDOSO, R.M. et al. Período de lactação e produção de leite, gordura e proteína, ajustados para 305 dias de lactação em vacas mestiças Europeu x Zebu. Revista da Sociedade Brasileira de Zootecnia, v.23, n.2, p.274-282, 1994.

TOUCHBERRY, R.W.; ROTTENSTEN, K.; ANDERSEN, H. Associations between service interval, interval from first service to conception, number of services per conception, and level of butterfat production. Journal of Dairy Science, v.42, p.1157-1170, 1959. 